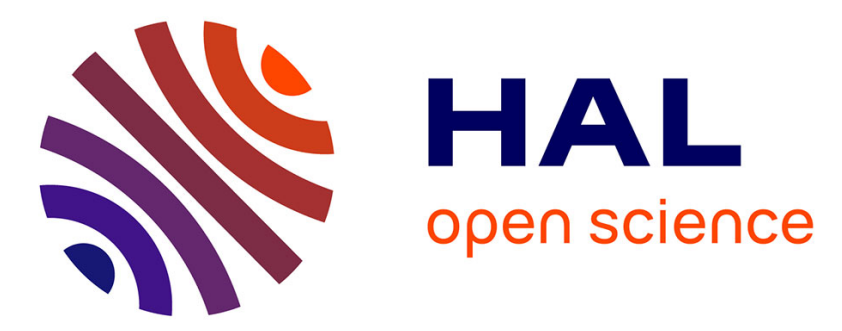

\title{
Continuous-Discrete High-Gain Extended Kalman Filter for Mobile Robots with Asynchronous Outputs
}

Aïda Feddaoui-Papin, Nicolas Boizot, Eric Busvelle, Vincent Hugel

\section{To cite this version:}

Aïda Feddaoui-Papin, Nicolas Boizot, Eric Busvelle, Vincent Hugel. Continuous-Discrete High-Gain Extended Kalman Filter for Mobile Robots with Asynchronous Outputs. 2019 8th International Conference on Systems and Control (ICSC), Oct 2019, Marrakech, Morocco. hal-02150425v2

\section{HAL Id: hal-02150425 \\ https://hal-univ-tln.archives-ouvertes.fr/hal-02150425v2}

Submitted on 27 Aug 2019

HAL is a multi-disciplinary open access archive for the deposit and dissemination of scientific research documents, whether they are published or not. The documents may come from teaching and research institutions in France or abroad, or from public or private research centers.
L'archive ouverte pluridisciplinaire HAL, est destinée au dépôt et à la diffusion de documents scientifiques de niveau recherche, publiés ou non, émanant des établissements d'enseignement et de recherche français ou étrangers, des laboratoires publics ou privés. 


\title{
Continuous-Discrete High-Gain Extended Kalman Filter for Mobile Robots with Asynchronous Outputs
}

\author{
Aïda Feddaoui-Papin ${ }^{1}$, Nicolas Boizot ${ }^{2}$, Eric Busvelle ${ }^{3}$ and Vincent Hugel ${ }^{4}$ \\ ${ }^{1}$ Aïda Feddaoui-Papin is with Laboratoire d'Informatique et des Systèmes, Université de Toulon, Aix Marseille \\ Univ, CNRS UMR 7020, LIS, Marseille, France, and with Laboratoire Conception des Systèmes Mécaniques et \\ Robotiques - COSMER EA 7398, Université de Toulon, France aida.feddaoui@univ-tln.fr \\ ${ }^{2}$ Nicolas Boizot is with Laboratoire d'Informatique et des Systèmes, Université de Toulon, Aix Marseille Univ, \\ CNRS UMR 7020, LIS, Marseille, France nicolas.boizot@univ-tln.fr \\ ${ }^{3}$ Eric Busvelle is with Laboratoire d'Informatique et des Systèmes, Université de Toulon, Aix Marseille Univ, \\ CNRS UMR 7020, LIS, Marseille, France eric.busvelle@univ-tln.fr \\ ${ }^{4}$ Vincent Hugel is with Laboratoire Conception des Systèmes Mécaniques et Robotiques - COSMER EA 7398, \\ Université de Toulon, France vincent.hugel@univ-tln.fr
}

This work was partially supported by the interdisciplinary program "Information, Numérique, Prévention" of the University of Toulon

\begin{abstract}
This paper details the implementation of an asynchronous high-gain Kalman filter on a mobile robot test platform on a path reconstruction problem. The observer algorithm, which was theoretically studied in a previous paper, is tailored to fit the problem at hand. Two path reconstruction experiments are carried out, using respectively a predefined trajectory and a joystick controlled trajectory. The estimation results are presented after offline processing. They show that our algorithm can be applied with success in the field of mobile robot navigation in the presence of asynchronous measurements.
\end{abstract}

\section{INTRODUCTION}

The last few years have seen a growing interest in observers for systems with asynchronous measurements. That is to say observers for systems having output variables not always available at the same time instants. To cite a few, articles $[1,2,3,4]$ propose such designs together with theoretical convergence analyses and illustrative examples, while case studies can be found in e.g. [5, 6, 7, 8, 9].

The present paper builds on [10] and [11] where the Kalman filter algorithm has been considered for continuous-discrete systems with asynchronous measurements. In particular, we proposed a definition of asynchronous systems that doesn't require any specific structure or regularity for the measurement procedure. On this basis, the global convergence of the observer has been demonstrated, in [10] for linear, observable, time-varying asynchronous systems, and in [11] for nonlinear systems, under appropriate observability assumptions, in the framework of high-gain observers.

In the following, we present an application of the latter observer to a path reconstruction problem for a nonholonomic mobile robot, the Turtlebot3 Waffle [12]. Indeed, in the experimental setting, measurements may not be reliable, due to either defective or low-quality sensors, undesirable obstacles or modelling errors. Therefore, the implementation of the observer is more challenging than in the simulation setting.

The rest of the article is organized as follows. First, the mathematical framework and problem under consideration are presented in Section 2. Then, the asynchronous setting and the observer are detailed in Section 3. Finally, experimental results are exposed in Section 4.

\section{Notations}

- w.r.t. stands for with respect to, and s.p.d. stands for symmetric positive definite. Oftentimes, time dependencies are omitted when there is no ambiguity.

- $\mathbb{O}_{(n \times m)}$ denotes the $(n \times m)$ empty matrix. 
- $I d_{n}$ denotes the n-dimensional identity matrix.

- A subdivision of time is a strictly increasing sequence of real numbers $\left\{\tau_{k}\right\}_{k \in \mathbb{N}}$ with $\tau_{0}=0$. A time-dependent quantity $x(t)$ evaluated at time $\tau_{k}$ is denoted $x_{k}=x\left(\tau_{k}\right)$.

- For an application $b(x, u), D_{x} b(x, y)$ is the Jacobian matrix computed w.r.t. to variable $x$.

- $A^{\prime}$ denotes the transpose of the matrix $A$.

\section{Modelling}

The Turtlebot3 Waffle mobile robot is controlled by means of two quantities: the linear and the angular velocities. In the following they are denoted by $v(\tau)$ and $u(\tau)$, respectively, and have to be understood as the control variables of the Reeds-Shepp car model:

$$
\left\{\begin{array}{l}
\dot{x}_{1}(\tau)=v(\tau) \cos (\gamma(\tau)) \\
\dot{x}_{2}(\tau)=v(\tau) \sin (\gamma(\tau)) \\
\dot{\gamma}(\tau)=u(\tau)
\end{array}\right.
$$

As it is schematised in Fig. 1a, let us consider an area delimited by two reflective beacons (denoted by $A$ and $B$ ) together with a reference frame attached to the center of beacon $A$. The x-axis of the reference frame is the straight line passing through the center of beacon $B$. The state of the mobile robot is $x=\left(x_{1}, x_{2}, \gamma\right)^{\prime} \in \mathbb{R}^{2} \times \mathbb{S}^{1}$, where:

- $\left(x_{1}, x_{2}\right)^{\prime}$ is the position of the middle point of the driving wheels axis;

- $\gamma$ is the robot's orientation, that is the angle formed by axes $\mathcal{X}_{1}^{r}$ and $\mathcal{X}_{1}^{b}$, cf. Fig. 1a.

The robot is equipped with a rotating $\operatorname{LIDAR}^{1}$ sensor positioned at point $\left(x_{1}, x_{2}\right)^{\prime}$. When the laser beam hits a reflective beacon, in other words, when it is aligned with the robot-beacon line ${ }^{2}$, this mechanism provides a measurement of the robot-beacon distance and of the angle formed by axis $\mathcal{X}_{1}^{b}$ and the robotbeacon line, see Fig. 1a. As in [11], the measurements of angles $\phi_{1}, \phi_{2}$ and distance $\rho_{1}$ are taken into account. The associated output model, where $x_{B}$ denotes the abscissa of $B$ in the reference frame, is:

$$
\left\{\begin{array}{l}
\phi_{1}(\tau)=\arctan \left(\frac{x_{2}}{x_{1}}\right)+\pi-\gamma \\
\rho_{1}(\tau)=\sqrt{\left(x_{1}^{2}+x_{2}^{2}\right)} \\
\phi_{2}(\tau)=\arctan \left(\frac{x_{2}}{x_{1}-x_{B}}\right)+\pi-\gamma
\end{array}\right.
$$

In order to make this latter set of equations easier to handle, system $\left(\Sigma_{R}\right)$ is rewritten using polar coordinates w.r.t. both $A$ and $B$-cf. Fig. 1b. Then, the angles $\alpha_{1}, \alpha_{2}$ and distances $\rho_{1}, \rho_{2}$ are such that:

- $x_{1}=\rho_{1} \cos \left(\alpha_{1}\right)$ and $x_{2}=\rho_{1} \sin \left(\alpha_{1}\right)$;

- $x_{1}=x_{B}+\rho_{2} \cos \left(\alpha_{2}\right)$ and $x_{2}=\rho_{2} \sin \left(\alpha_{2}\right)$.

In those new coordinates, the full dynamics, with output vector $y(t)$, are given by:

$$
\begin{gathered}
\left(\begin{array}{c}
\dot{\alpha}_{1} \\
\dot{\rho}_{1} \\
\dot{\alpha}_{2} \\
\dot{\rho}_{2} \\
\dot{\gamma}
\end{array}\right)=\left(\begin{array}{c}
\frac{v}{\rho_{1}} \sin \left(\gamma-\alpha_{1}\right) \\
v \cos \left(\gamma-\alpha_{1}\right) \\
v \\
\frac{v}{\rho_{2}} \sin \left(\gamma-\alpha_{2}\right) \\
v \cos \left(\gamma-\alpha_{2}\right) \\
u
\end{array}\right), \\
y(t)=\left(\begin{array}{c}
\phi_{1} \\
\rho_{1} \\
\phi_{2}
\end{array}\right)=\left(\begin{array}{c}
\pi+\alpha_{1}-\gamma \\
\rho_{1} \\
\pi+\alpha_{2}-\gamma
\end{array}\right) .
\end{gathered}
$$

\footnotetext{
${ }^{1}$ LIght Detection And Ranging.

${ }^{2}$ Robot-beacon line: the line that passes through $\left(x_{1}, x_{2}\right)^{\prime}$ and the center of the concerned beacon.
} 
With the help of system $\left(\Sigma_{P}\right)$, the original system $\left(\Sigma_{R}\right)$ is shown to be observable. Indeed, we can find a change of coordinates that puts $\left(\Sigma_{R}\right)$ under a normal observability form -i.e. system $\left(\Sigma_{N}\right)$ below. Actually, the exact position of the robot can be computed from the knowledge of $\phi_{2}-\phi_{1}$ and $\rho_{1}-$ which are the state variables of $\left(\Sigma_{N}\right)$. The orientation of the robot can then be easily deduced.

Let us define $z(\tau)=y(\tau)$ which yields to the following normal observability form:

$$
\left\{\begin{array}{l}
\dot{z}(\tau)=A(u) z(\tau)+b(z, u) \\
y(\tau)=C z(\tau)
\end{array}\right.
$$

where $A(u)=\mathbb{O}_{(3 \times 3)}, C(\tau)=I d_{3}$ and

$$
b(z(\tau), u(\tau))=\left(\begin{array}{c}
\frac{v \sin \left(z_{1}\right)}{z_{2}}-u \\
-v \cos \left(z_{1}\right) \\
\frac{v \sin \left(z_{3}\right)}{\bar{\rho}\left(z_{1}, z_{2}, z_{3}\right)}-u
\end{array}\right) .
$$

In the vector field (3), the mapping $\bar{\rho}\left(z_{1}, z_{2}, z_{3}\right)$ corresponds to $\rho_{2}$ obtained through Al-Kashi's law of cosines. It has the following form:

$$
\bar{\rho}\left(z_{1}, z_{2}, z_{3}\right)=z_{2} \cos \left(z_{3}-z_{1}\right)+\sqrt{x_{B}^{2}-z_{2}^{2}} \sin ^{2}\left(z_{3}-z_{1}\right)
$$

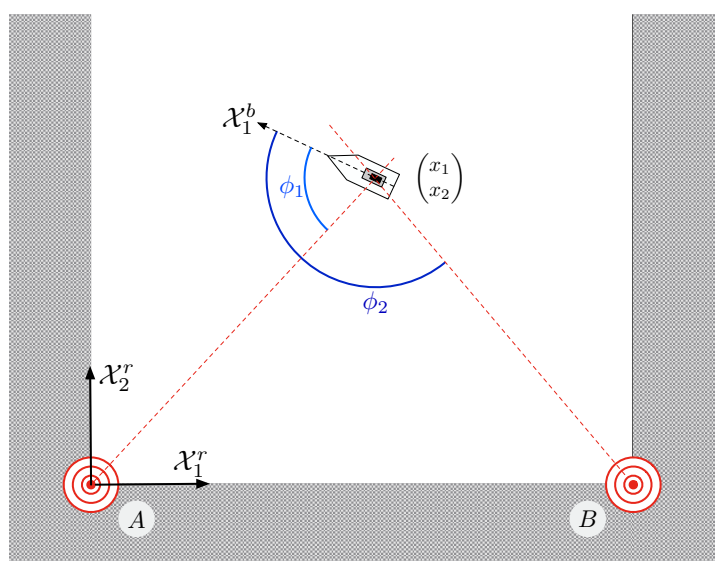

(a) Notations (planar representation).

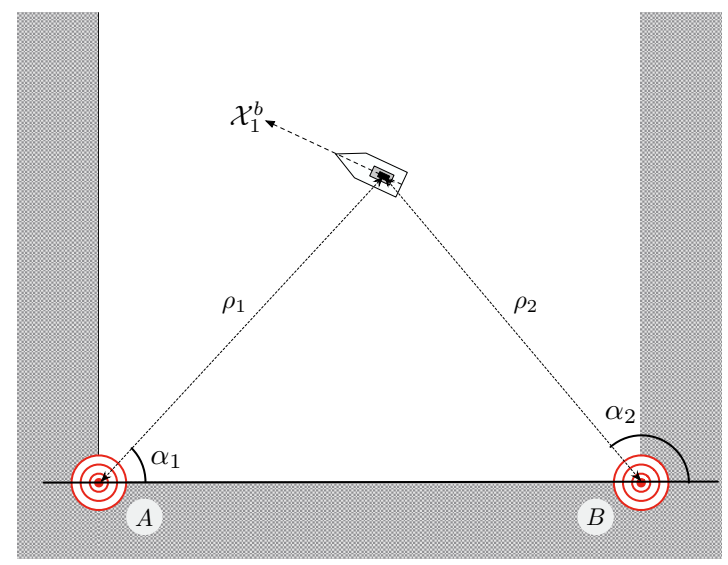

(b) Notations (polar representations).

Figure 1: Geometry

\section{Asynchronous High-gain EKF}

The robot's physical sensor, a rotating LIDAR, cannot provide continuous measurements of the output vector $y(\tau)$. Moreover, all those measurements are neither synchronous nor uniformly taken. Therefore, system $\left(\Sigma_{N}\right)$ is not a faithful model of the robot's dynamics. As such, following the formalism proposed in [11], this section presents an asynchronous version of $\left(\Sigma_{N}\right)$ together with the ad hoc high-gain extended Kalman filter. In other words, the general context of the above cited article is adapted to the system $\left(\Sigma_{N}\right)$ in terms of state space and sensor output variables.

Two sets of measurements are available: $y_{1}$ and $y_{2}$ on the one hand, and $y_{3}$ on the other hand. Accordingly, we define two numerical sensors - denoted by $\left(\mathfrak{s}_{1}\right)$ and $\left(\mathfrak{s}_{2}\right)$ - and the output vectors:

$$
\begin{cases}y^{\left(\mathfrak{s}_{1}\right)}(\tau)= & \left(\begin{array}{l}
y_{1}(\tau) \\
y_{2}(\tau)
\end{array}\right)=C_{1} z(\tau) \\
y^{\left(\mathfrak{s}_{2}\right)}(\tau)=\left(y_{3}(\tau)\right)=C_{2} z(\tau)\end{cases}
$$


where matrices $C_{1}$ and $C_{2}$ are of the form:

$$
C_{1}=\left(\begin{array}{lll}
1 & 0 & 0 \\
0 & 1 & 0
\end{array}\right) \quad \text { and } \quad C_{2}=\left(\begin{array}{lll}
0 & 0 & 1
\end{array}\right) .
$$

For $i \in\{1,2\}$, a subdivision of time $\left\{s_{k}^{(i)}\right\}_{k \in \mathbb{N}}$ is associated to sensor $\left(\mathfrak{s}_{i}\right)$, and a global subdivision $\left\{\tau_{k}\right\}_{k \in \mathbb{N}}$ is defined as:

$$
\left\{\tau_{k}\right\}_{k \in \mathbb{N}}:=\left\{s_{l}^{(1)}\right\}_{l \in \mathbb{N}} \cup\left\{s_{l}^{(2)}\right\}_{l \in \mathbb{N}}
$$

This is well defined in the physical space — when the line $A B$ is not accessible - since $\left\{s_{l}^{(1)}\right\}_{l \in \mathbb{N}} \cap$ $\left\{s_{l}^{(2)}\right\}_{l \in \mathbb{N}}=\emptyset$. At each time $\tau_{k}$, it is assumed that one of the two sensors $\left(\mathfrak{s}_{i}\right)$ is available and there exists an index $l$ such that $s_{l}^{(i)}=\tau_{k}$. Although the measurement procedure implies that numerical sensors readings should take place alternatively, in practice, misreadings might occur. In such cases, corresponding readings are dropped. Thus, the assumption of alternative readings cannot be guaranteed. As a consequence, no specific structure can be expected from the $\left\{\tau_{k}\right\}_{k \in \mathbb{N}}$ subdivision, and the index $l$ such that $s_{l}^{(i)}=\tau_{k}$ is denoted $l_{k}^{(i)}$.

Let $\tau_{k}$ be a measurement time. We define $\sigma_{k}$ as being equal to 1 if there is an index $l_{k}^{(1)}$ such that $s_{l_{k}^{(1)}}^{(1)}=\tau_{k}$. Alternatively, $\sigma_{k}$ equals 2 if $\tau_{k} \in\left\{s_{l}^{(2)}\right\}_{l \in \mathbb{N}}$.

The asynchronous continuous-discrete version of $\left(\Sigma_{N}\right)$ is:

$$
\left\{\begin{array}{l}
\dot{z}(\tau)=A(u(\tau)) z(\tau)+b(z(\tau), u(\tau)) \\
y\left(\tau_{k}\right)=C_{\sigma_{k}} z\left(\tau_{k}\right)
\end{array}\right.
$$

Let us now describe the asynchronous high-gain extended Kalman filter associated to $\left(\Sigma_{A N}\right)$. It is defined in two parts:

1. two prediction equations when $\tau \in\left[\tau_{k-1}, \tau_{k}\left[, k \in \mathbb{N}^{*}\right.\right.$, with initial values $\hat{z}_{k-1}^{(+)}$and $S_{k-1}^{(+)}$;

2. two correction equations at time $\tau_{k}$.

\section{Notations:}

- $\hat{z}$ is the estimated state and $S$ is the Ricatti matrix (the notations below are also used for $S$ );

- $\hat{z}(\tau)$ is the estimated state for all $\tau \in\left[\tau_{k-1}, \tau_{k}[\right.$;

- $\hat{z}_{k}^{(-)}$is the estimated state at time $\tau_{k}$, at the end of a prediction step and before a correction step;

- $\hat{z}_{k}^{(+)}$is the estimated state at time $\tau_{k}$, after a correction step. Therefore, $\hat{z}_{k}^{(+)}$is the initial estimated state of the new prediction interval $\left[\tau_{k}, \tau_{k+1}[\right.$.

- $S(0)$ is a $(3 \times 3)$ s.p.d. real matrix;

- $Q$ is a $(3 \times 3)$ s.p.d. real matrix;

- $R^{(1)}$ a $(2 \times 2)$ s.p.d. real matrix and $R^{(2)}>0$ is a scalar;

- $\theta>1$ denotes the so called (fixed) high-gain parameter;

- finally, $Q_{\theta}=\theta Q$ and $R_{\theta}^{\left(\sigma_{k}\right)}=\frac{1}{\theta} R^{\left(\sigma_{k}\right)}$.

\section{Prédiction Equations}

$$
\left\{\begin{array}{rr}
\dot{\hat{z}}(\tau)= & A(u) \hat{z}+b(\hat{z}, u) \\
\dot{S}(\tau)= & -\left[A(u)+D_{z} b(\hat{z}, u)\right]^{\prime} S \\
& \quad-S\left[A(u)+D_{z} b(\hat{z}, u)\right]-S Q_{\theta} S
\end{array}\right.
$$




\section{Corrections Equations}

$$
\left\{\begin{array}{cc}
\hat{z}_{k}^{(+)}= & \hat{z}_{k}^{(-)}-S_{k}^{(+)^{-1}} C_{\sigma_{k}}^{\prime}\left(R_{\theta}^{\left(\sigma_{k}\right)}\right)^{-1} \times \\
& \left(C_{\sigma_{k}} \hat{z}_{k}^{(-)}-y_{k}\right)\left(s_{l_{k}^{(i)}}^{(i)}-s_{l_{k}^{(i)}-1}^{(i)}\right) \\
S_{k}^{(+)}= & S_{k}^{(-)}+C_{\sigma_{k}}^{\prime}\left(R_{\theta}^{\left(\sigma_{k}\right)}\right)^{-1} C_{\sigma_{k}}\left(s_{l_{k}^{(i)}}^{(i)}-s_{l_{k}^{(i)}-1}^{(i)}\right)
\end{array}\right.
$$

In other words, the correction at a time $\tau_{k}$ is performed with respect to each measurement $y_{k}=$ $C_{\sigma_{k}} z(t)$ that is actually available. It also involves the weighting factor $\left(s_{l_{k}^{(i)}}^{(i)}-s_{l_{k}^{(i)}-1}^{(i)}\right)$ equal to the time elapsed since the last time this specific output was measured.

For a thorough definition of this observer, and a complete exposure of its proof of convergence, interested readers may refer to [11].

\section{Experimental Results}

\subsection{Equipment}

The Turtlebot3 Waffle is a small mobile robot produced by Robotis [12] whose dimensions are given in Fig. 2. It is endowed with an Intel ${ }^{\circledR}$ Joule $^{\mathrm{TM}} 570 \mathrm{x}$ single board computer, and a 32-bit ARM Cortex ${ }^{\circledR}$ M7 microcontroller unit. Measurements obtained from a rotating LIDAR sensor are used to implement the observer algorithm, and odometry sensors provide ground truth data. Finally, the robot is operated via the ROS middleware [13]. Commands are sent by means of Python scripts that interpret either joystick generated or preset instructions. For each experiment, LIDAR, commands and odometry data are recorded in a log file. This file is then converted into CSV files which are processed by the observer's code.

The rotating LIDAR sensor is a RPLIDAR A2 from Slamtec [14] which was placed at the middle point of the driving wheels axis ${ }^{3}$. The accuracy of this LIDAR was assessed through a series of experiments using different types of beacons. A mean bias of $1 \mathrm{~cm}$ with a variance of $1.5 \mathrm{~cm}$ was recorded on distance measurements.

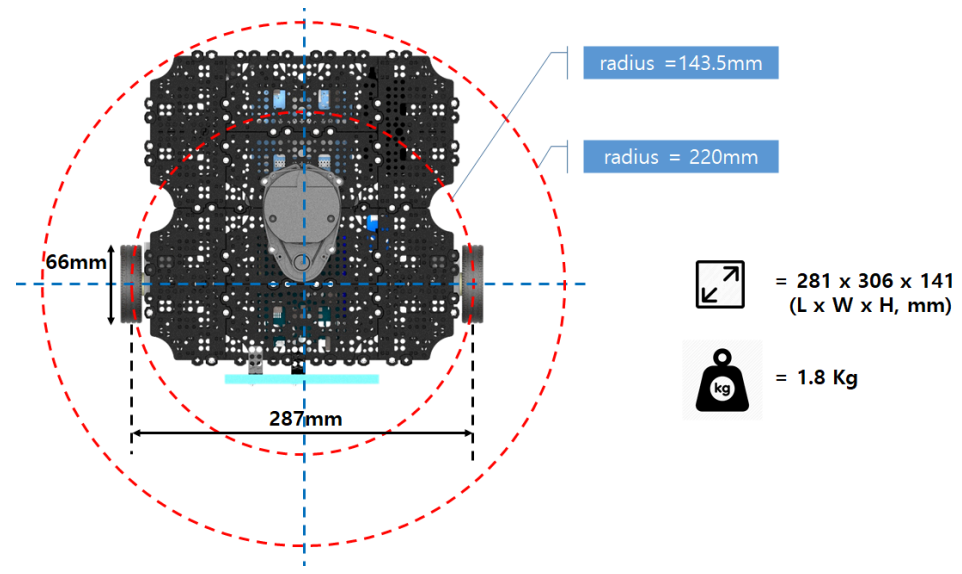

Figure 2: Turtlebot3 Waffle (source: [12])

\subsection{Ground Truth}

The ground truth is provided by the odometry data which was validated with the help of a webcam filming from above. After a calibration step based on the pinhole model, the robot trajectory is extracted from video data. For a 82 centimeters square trajectory, the two graphs obtained from video and odometry data remain into a $0.5 \mathrm{~cm}$ wide band.

\footnotetext{
${ }^{3}$ On Fig. 2, it is the intersection between the two blue dotted lines.
} 


\subsection{Beacons}

We used two J11 beacons of 19.9 centimeters in diameter having a reflective strip placed at an appropriate height. Since the LIDAR has a resolution of 1 degree, 359 distance measurements are taken during a LIDAR rotation. When the sensor scans a beacon, a number of measurements relating to it are taken, and this number may vary from one rotation to another. If the robot remained still, those successive measurements of a beacon would form a circular arc. Although the robot is in motion, we suppose that this circular arc is not distorted much since the velocity of the robot is low. Therefore, a curve fitting algorithm is used to reconstruct the center of a beacon, to determine the corresponding angle and to label the measurement. Angle values that are not consistent with previous detections are dropped and interpreted as misreadings. This approach is more robust w.r.t. measurement noise than our first attempts which consisted in using the nearest point or the mean angle formed by the robot with a beacon. It also allows us to discriminate beacons from artefacts, which is illustrated in Fig. 3 and Fig. 4. Each figure contains two graphs, the top one shows angle measurements and the bottom one, distance measurements. When a detected object is determined to be a beacon, it is marked using a cross symbol - in red for beacon A, blue for beacon B-, while a black dot marks a false positive.

Let us note that the experiments presented in this paper were performed under laboratory conditions. Therefore, it was easy to label the beacons since we knew which one was detected first. However, without this knowledge, the technique used to recover the center of a given beacon can be used to discriminate between two beacons provided they are chosen with sufficiently different diameters.
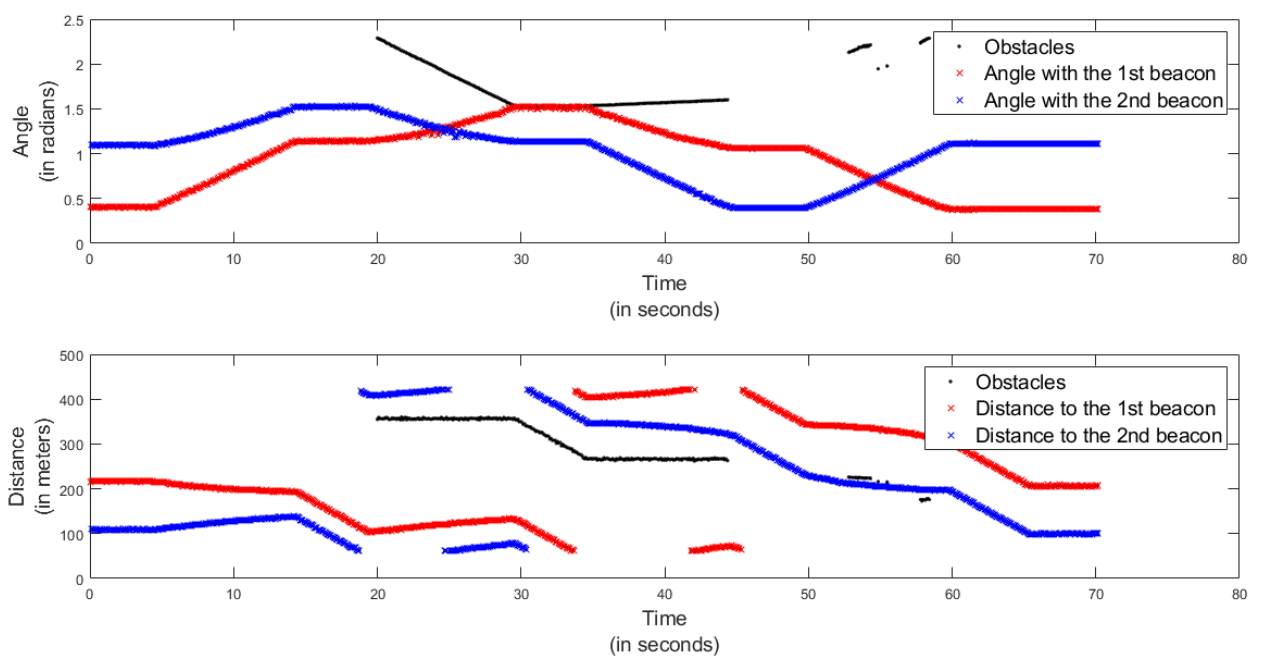

Figure 3: Output measurements of the first experiment - i.e. using preset instructions. Black dots correspond to false positive.

\subsection{Experiments}

As stated in the abstract, two different experiments were performed. In the first one, the robot followed preset open loop instructions meant to describe a square-like trajectory -i.e. the black dashed line in Fig. 5-, with a linear velocity of 0.08 meter/second and an angular velocity of $0.316 \mathrm{radian} / \mathrm{second}$. In the second experiment, the robot was operated by means of a remote controller, which led to the erratic trajectory represented by a black dashed line in Fig. 7, with a maximal linear velocity of 0.17 meter/second and a maximal angular velocity of 1.259 radian/second. In order to provide a better understanding of this trajectory, the physical -i.e. cartesian- coordinates are plotted against time in Fig. 8.

The observer parameters were empirically set to the following values:

- $\theta=2.5$ for the first experiment, $\theta=1.5$ for the second one;

- $Q=10^{-2} I d_{3}$;

- $R^{(1)}=I d_{2}$, and $R^{(2)}=1$; 

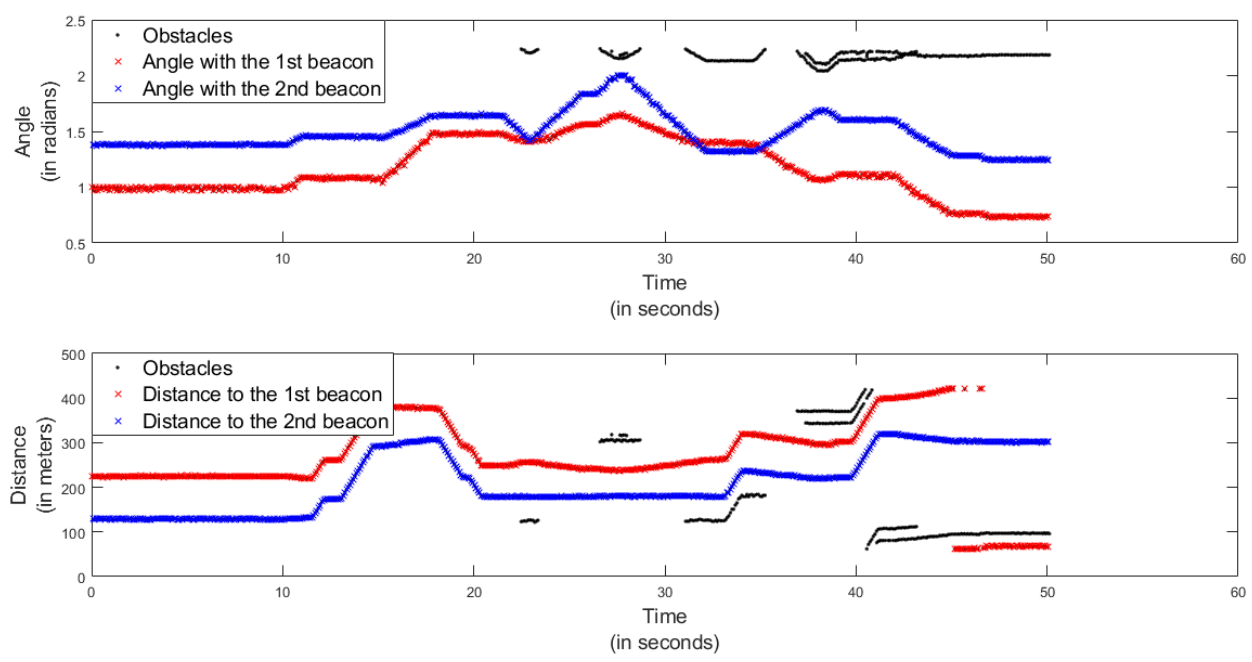

Figure 4: Output measurements of the second experiment -i.e. using joystick generated instructions. Black dots correspond to false positive.

- the initial Ricatti matrix $S_{0}$ has been chosen as the solution of the static Ricatti equation solved using time zero data.

The estimated trajectories in the physical space are shown in red in Fig. 5 and Fig. 7. Also, Fig. 6 and Fig. 9 highlight the error between the estimated state and the ground truth data in normal coordinates.

In order to provide the reader with a better understanding of what happens during the first experiment, the estimated position and the corresponding odometry data are highlighted at three different time instants. Markers having the same colour correspond to a same time instant — filled star symbols are used to mark estimated positions while empty star symbols are used on the odometry graph. Those time instants are also pointed out by the three vertical lines that appear on Fig. 6:

- at time $t=9.9 \mathrm{~s}$ the robot starts its first rotation;

- time $t=15 \mathrm{~s}$ corresponds to the end of the first rotation;

- the markers at approximately $25 \mathrm{~s}$ correspond to the beginning of the second rotation.

\section{CONCLUSIONS}

The high-gain observer approach is a powerfull way to construct exponentially converging observers. Nevertheless, a classical drawback is their sensitivity to noise. Approaches based on the high-gain extended Kalman filter provide a solution to this problem since it inherits from extended Kalman filter its capacity to tackle noise. Although it is also possible to adapt online high-gain parameter to innovation - and hence to the error [15] —, it was not needed in this practical case. Furthermore, this application illustrates the ability of high-gain extended Kalman filter to use the dynamics of the system in order to reconstruct information from asynchronous measurements. Indeed, the position of the moving robot may be estimated only from measurements and from the state equation which relates each measurements. Therefore, the change of coordinates and the high-gain parameter are respectful of the robot's model. We consider that this remark deserves to be made since the classical proof of convergence of highgain observers consists in neglecting dynamics beside measurements, thanks to the parameter $\theta$. This experiment and the proof of observability with asynchronous measurements illustrate that the main dynamical aspects of the process are preserved.

Some improvements are left for the future. First of all, it would be interesting to compare the results of this experiment with extensions of the study to the case where outputs are $\phi_{1}, \phi_{2}$ and $\rho_{2}$, with $\rho_{2}$ the distance of the robot to beacon $B$ and the case with the four outputs $\phi_{1}, \rho_{1}$ and $\phi_{2}, \rho_{2}$. The underlying idea is to improve the algorithm by switching those three sets of outputs on the basis of a criterion assessing the accuracy of the estimated trajectory or the reliability of a given measurement. 


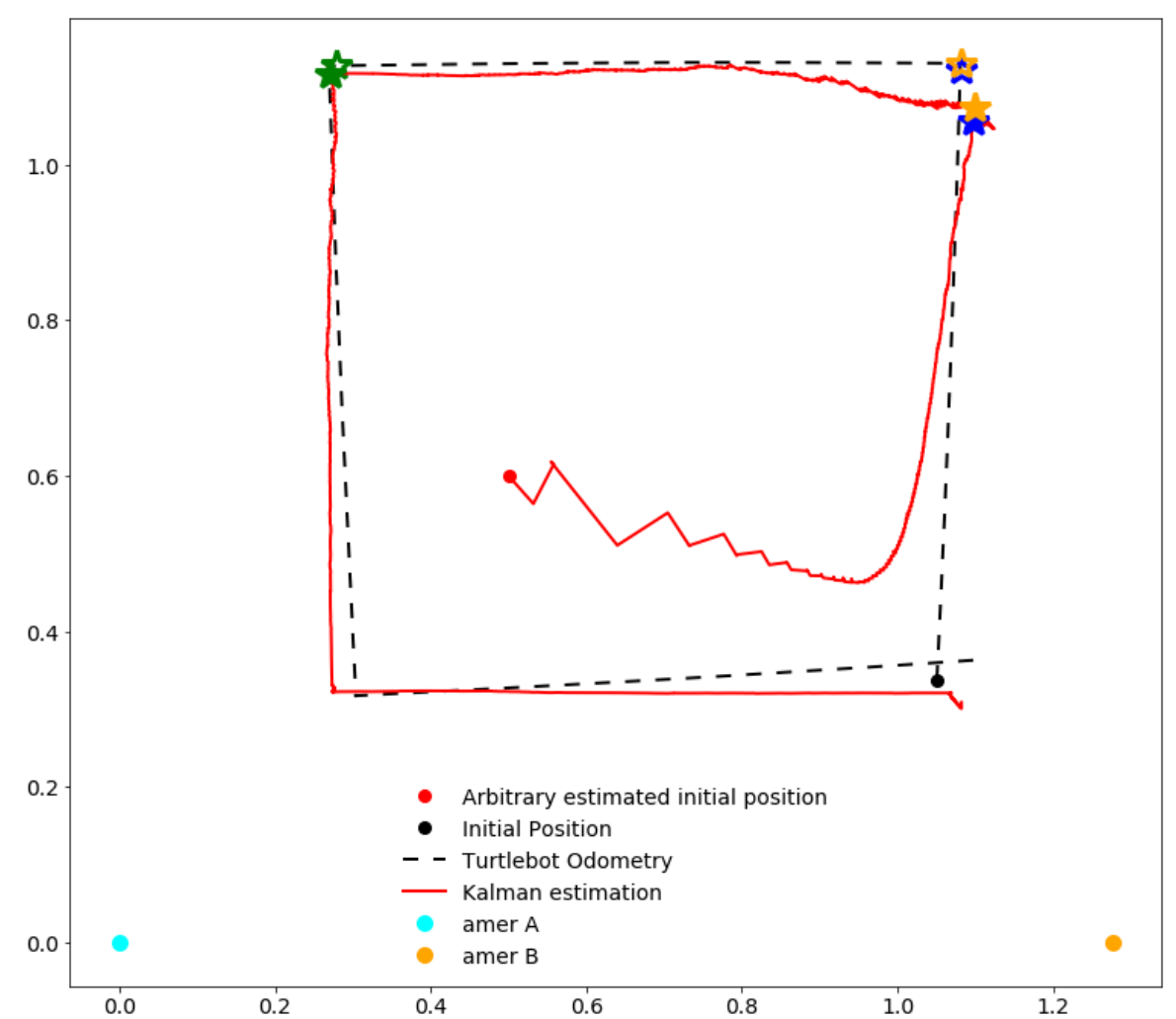

Figure 5: Movement with preset instructions: representation in the phase space
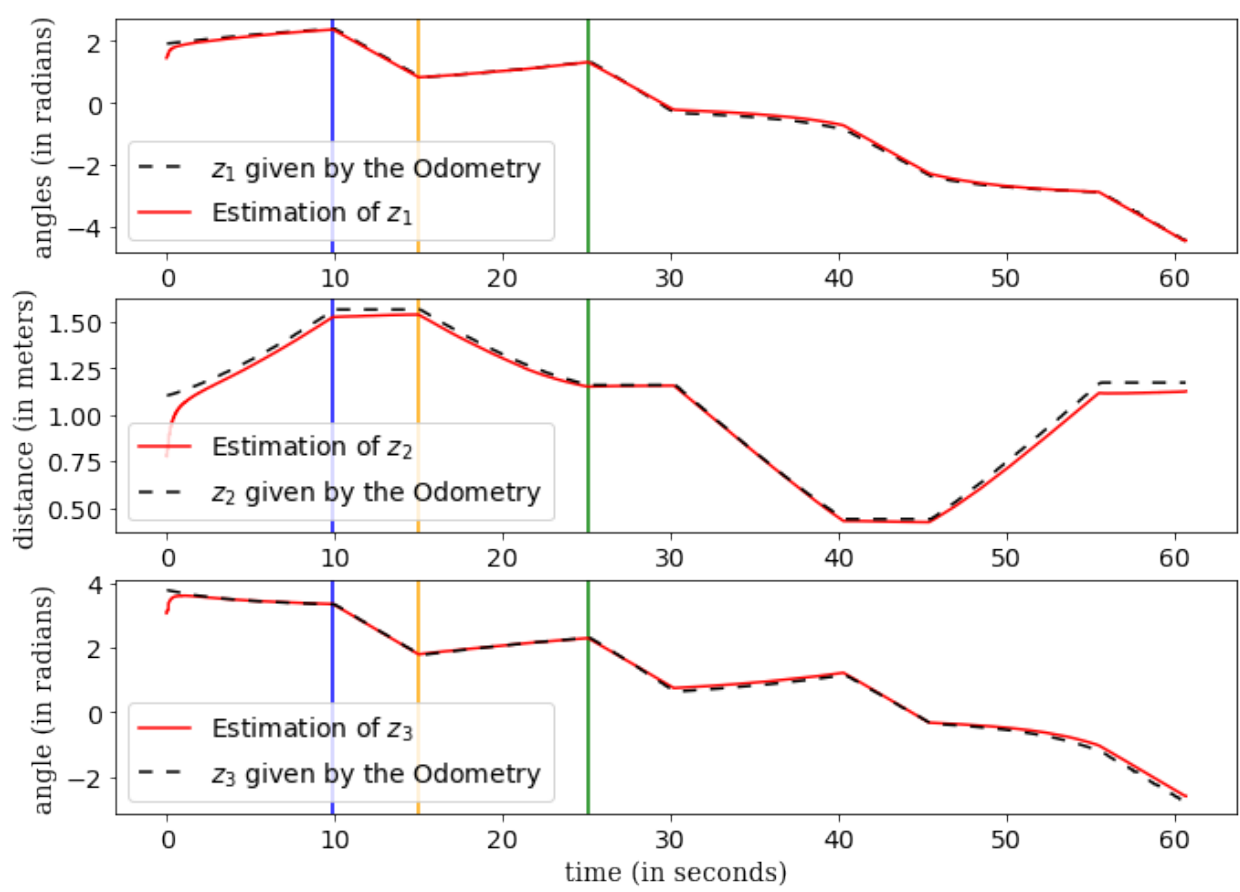

Figure 6: Movement with preset instructions: representation in the normal coordinates

Finally, we plan to test this observer on an underwater robot. The difficulty will be to move from a two-dimensional environment with three state variables (position in $\mathbb{R}^{2}$ and orientation) to a threedimensional environment with six state variables (position in $\mathbb{R}^{3}$, roll, pitch, yaw) and manage different 


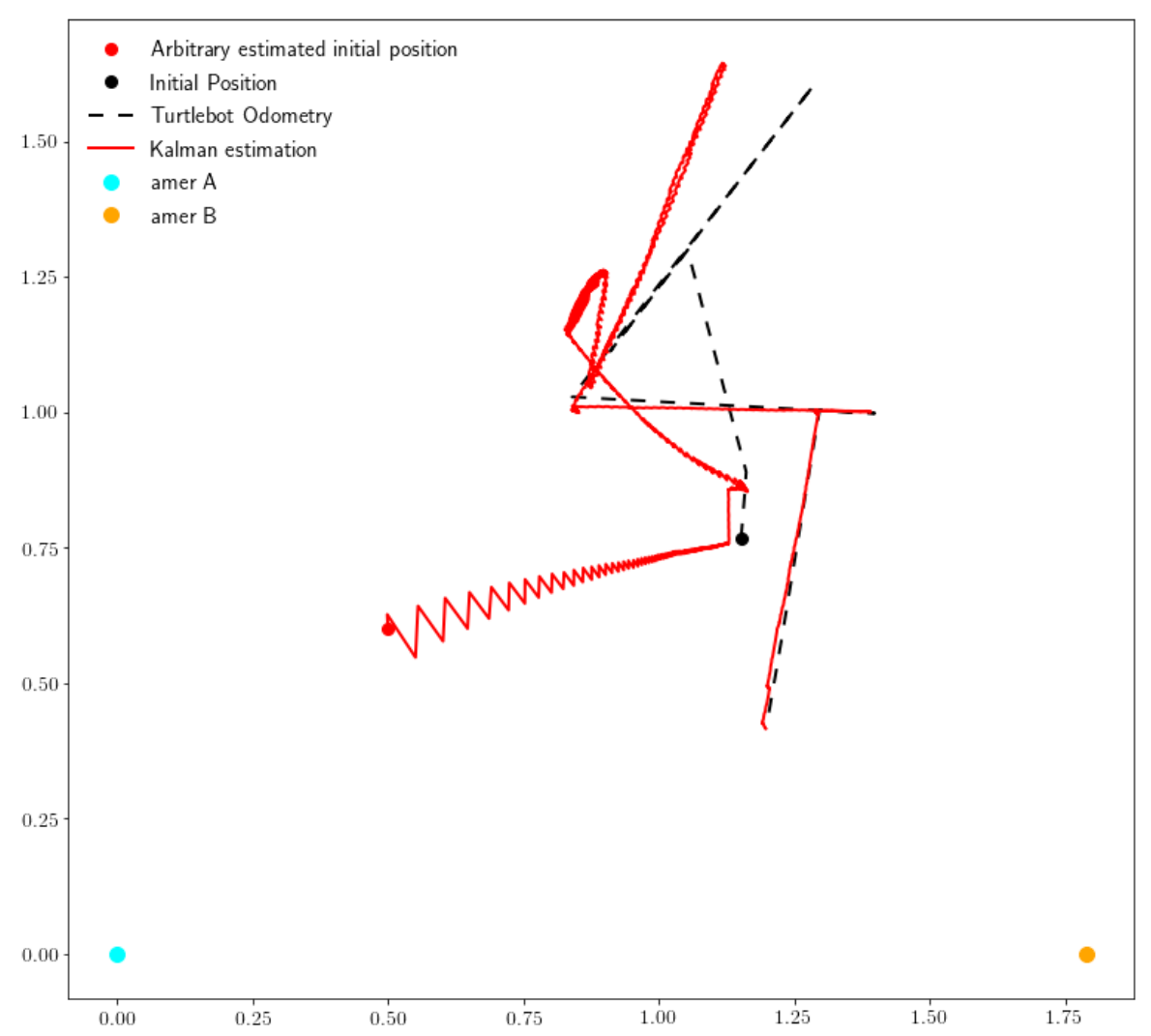

Figure 7: Movement with a joystick: representation in the phase space
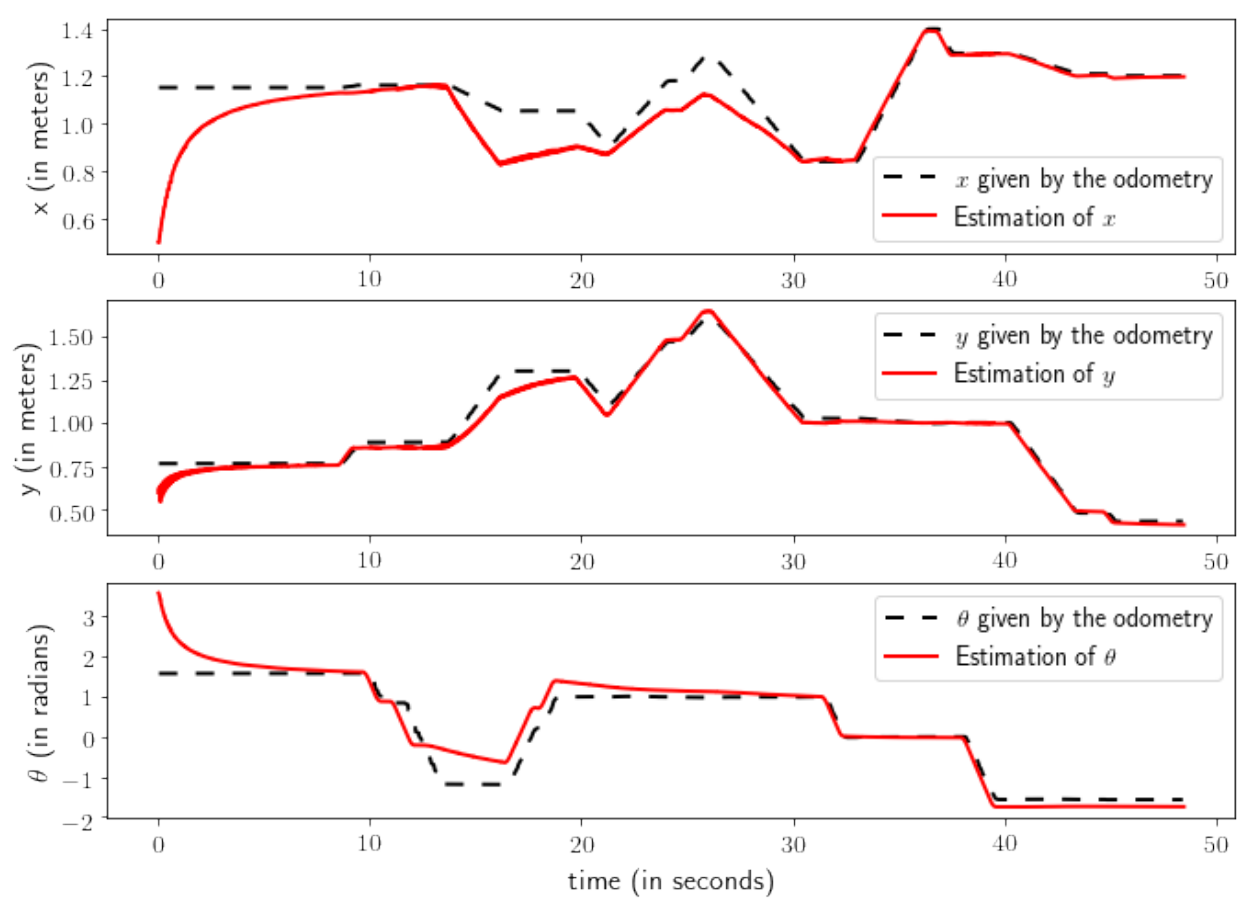

Figure 8: Movement with a joystick: representation in the cartesian coordinates

sensors - the ground truth can no more be obtained using the odometry sensors. 


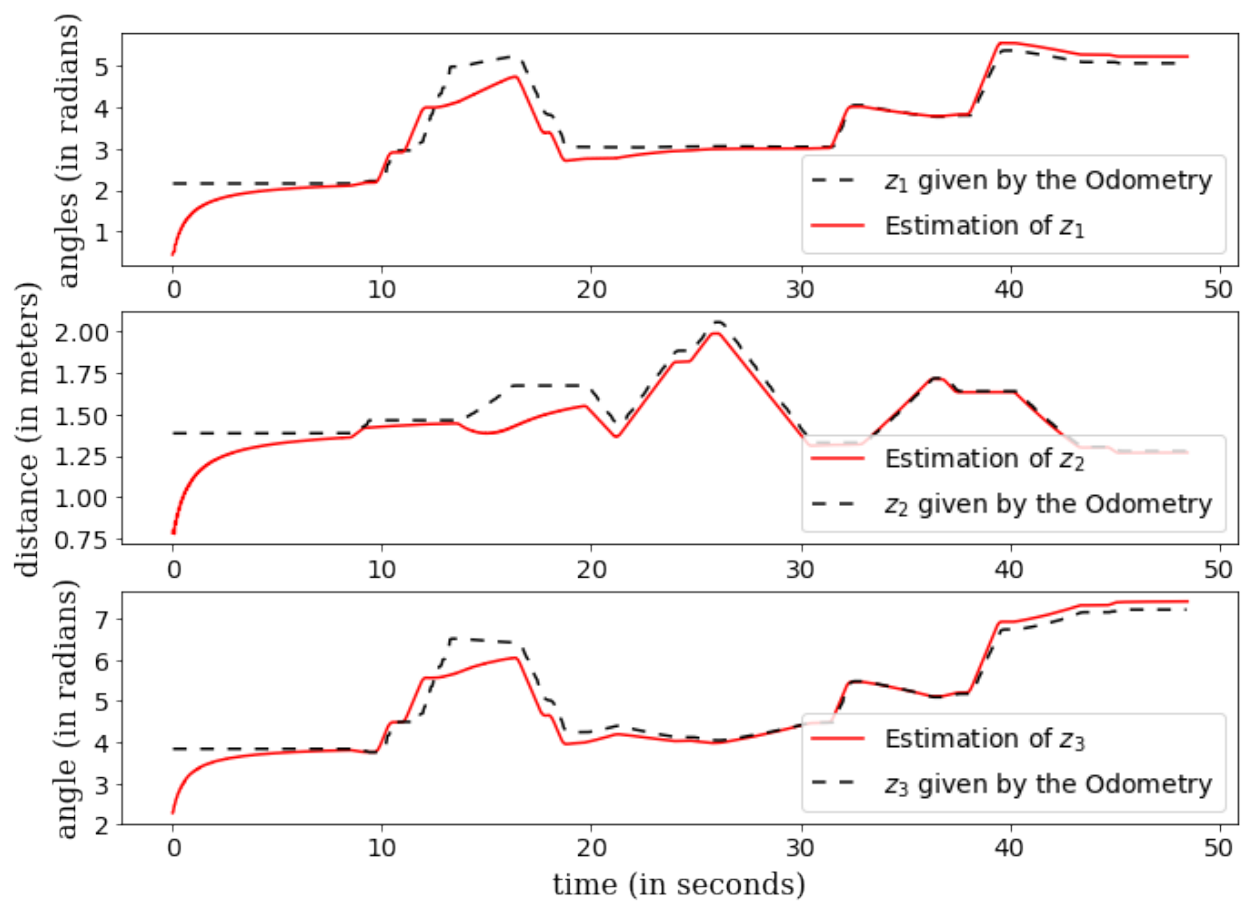

Figure 9: Movement with a joystick: representation in the normal coordinates

\section{ACKNOWLEDGMENT}

We are grateful to Albane Ordrenneau and Adrien Mojica for their involvement during their master's internship and the help they provided for the handling of the robot and the data processing. We also thank Claire Dune, from COSMER, for all her advice. Finally, we have a thought for the foreman of the Maintenance Pole of the University of Toulon who provided us with the J11 beacons. 


\section{References}

[1] W. Cao and Y. C. Soh, "Nonlinear multi-rate current state estimation: convergence analysis and application to biological systems," Computers and Chemical Engineering, vol. 28, pp. 1623-1633, 2004.

[2] M. Moarref and L. Rodrigues, "Observer design for linear multi-rate sampled-data systems," in Proc. American Control Conference (ACC), 2014, pp. 5319-5324.

[3] C. Ling and C. Kravaris, "Multi-rate observer design for process monitoring using asynchronous inter-sample output predictions," AIChE Journal, vol. 63, no. 8, pp. 3384-3397, 2017.

[4] — - "Multi-rate sampled-data observers based on a continuous-time design," in Proceedings of the 56th IEEE Conference on Decision and Control (CDC), 2017, pp. 3664-3669.

[5] E. A. Butcher and J. Wang, "On kalman filtering and observability in nonlinear sequential relative orbit estimation," Journal of Guidance, Control, and Dynamics, vol. 40, no. 9, pp. 2167-2182, 2017.

[6] P.-J. Bristeau, E. Dorveaux, D. Vissière, and N. Petit, "Hardware and software architecture for state estimation on an experimental low-cost small-scaled helicopter," Control Engineering Practice, vol. 18, no. 7, pp. 733-746, 2010.

[7] R. D. Gudi, S. L. Shah, and M. R. Gray, "Adaptive multirate state and parameter estimation strategies with applications to a bioreactor," AIChE Journal, vol. 41, no. 11, pp. 2451-2464, 1995.

[8] D. Mayhew and P. Kachroo, "Multirate sensor fusion for GPS using Kalman filtering, fuzzy methods, and map matching," in Proceedings of SPIE: Mobile Robots XIII and Intelligent Transportation Systems, vol. 3525(1), 1998, pp. 440-449.

[9] H. Zhang, M. V. Basin, and M. Skliar, "Itô-volterra optimal state estimation with continuous, multirate, randomly sampled, and delayed measurements," IEEE Transactions on Automatic Control, vol. 52, no. 3, pp. 401-416, 2007.

[10] A. A. Feddaoui, N. Boizot, E. Busvelle, and V. Hugel, "A Kalman filter for linear continuous-discrete systems with asynchronous measurements," in Proceedings of the 56th IEEE Conference on Decision and Control (CDC), 2017, pp. 2813-2818.

[11] A. Feddaoui, N. Boizot, E. Busvelle, and V. Hugel, "High-gain extended Kalman filter for continuous-discret systems with asynchronous measurements," International Journal of Control (DOI: 10.1080/00207179.2018.1539525), 2018.

[12] (2019). [Online]. Available: emanual.robotis.com

[13] M. Quigley, B. Gerkey, and W. D. Smart, Programming Robots with ROS. O' Reilly, 2015.

[14] (2019). [Online]. Available: www.slamtec.com

[15] N. Boizot, E. Busvelle, and J.-P. Gauthier, "An adaptive high-gain observer for nonlinear systems," Automatica, vol. 46, no. 9, pp. 1483-1488, 2010. 